\title{
Barreiras de acesso relacionadas ao processo de prescrição e dispensação de medicamentos em uma Unidade Básica de Saúde do Distrito Federal
}

\author{
Access barriers related to the process of prescription and dispense of medications in a \\ Basic Health Unit in the Federal District
}
Barreras de acceso relacionadas con el proceso de prescripción y dispensación de medicamentos en una Unidad Básica de Salud del Distrito Federal

\begin{abstract}
Marina Fagundes Moreira da Silva ${ }^{1 *}$, Alexandre Vaz Machado, Débora Santos Lula Barros².
\end{abstract}
\begin{abstract}
RESUMO
Objetivo: Identificar as principais causas de demanda reprimida referentes ao acesso a medicamentos, considerando as barreiras documentais e/ou legais, em uma Unidade Básica de Saúde (UBS) do Distrito Federal, Brasil. Métodos: Para tanto, foi realizado um estudo transversal e observacional. Os dados foram coletados, de forma presencial, nos meses de julho e agosto de 2020. Para a definição dos critérios de identificação das prescrições em que não houve acesso pelos usuários do SUS foram considerados os parâmetros definidos na Portaria $n^{\circ}$ 250, de 17 de Dezembro de 2014 da Secretaria de Estado de Saúde do Distrito Federal. Resultados: Foram identificados 277 fatores inviabilizantes em 254 prescrições. A categoria "ausência de documentos necessários" foi responsável por 6,14\% do não fornecimento; "ausência de duas vias" por 14,08\%; "em desacordo com a Denominação Comum Brasileira" por 9,75\%; "informações incompletas, ilegíveis ou caracterização de emendas e rasuras" por 5,42\%; "não disponibilizados" por 49,82\%. Conclusão: Os dados apresentados indicam que existe um problema relacionado a questões técnicas e burocráticas referentes as prescrições que interferem no acesso a medicamentos.
\end{abstract}

Palavras-chave: Assistência farmacêutica, Medicamentos essenciais, Acesso aos serviços de saúde, Atenção primária à saúde, Sistema único de saúde.

\begin{abstract}
Objective: Identify the main causes of repressed demand of access to medicines considering legal barriers in a Basic Health Unit in the Federal District, Brazil. Methods: A cross-sectional and observational study was carried out. The data were collected in person in the months of July and August 2020. In order to define the criteria for identifying the prescriptions in which there was no access by SUS users was considered the parameters defined in Ordinance number 250 of 17 December 2014 from the Health Department of the Federal District. Results: 277 unviable factors were identified in 254 prescriptions. The category "absence of necessary documents" was responsible for $6.14 \%$ of non-supply; "absence of two ways" by $14.08 \%$; "in disagreement with the Brazilian Common Denomination" by $9.75 \%$; "incomplete, illegible information or characterization of amendments and erasures" by $5.42 \%$; "not available" by $49.82 \%$. Conclusion: The results indicate that there is a problem related to technical and bureaucratic issues regarding prescriptions that interfere with access to medicines.
\end{abstract}

Keywords: Pharmaceutical services, Drugs essential, Health services accessibility, Primary health care, Unified health system.

\footnotetext{
${ }^{1}$ Fundação de Ensino e Pesquisa em Ciências da Saúde (FEPECS), Brasília - DF.

*E-mail: marinafagundesms@gmail.com

${ }^{2}$ Secretaria de Saúde do Distrito Federal, Brasília - DF.
} 


\section{RESUMEN}

Objetivo: Identificar las principales causas de demanda reprimida en materia de acceso a medicamentos, considerando las barreras documentales y / o legales, en una Unidad Básica de Salud (UBS) en el Distrito Federal, Brasil. Métodos: Se realizó un estudio transversal y observacional. Los datos fueron recogidos, presencialmente, en los meses de julio y agosto de 2020. Para definir los criterios para identificar las recetas en que no hubo acceso por parte de los usuarios del SUS, se consideraron los parámetros definidos en la Ordenanza No. 250, de 17 de diciembre de 2014 del Departamento de Salud del Estado del Distrito Federal. Resultados: Se identificaron 277 factores inviables en 254 recetas. La categoría "ausencia de documentos necesarios" fue responsable del $6,14 \%$ de la falta de suministro; "Ausencia de dos vías" en un 14,08\%; "En desacuerdo con la Denominación Común Brasileña" en un 9,75\%; "Información incompleta, ilegible o caracterización de enmiendas y tachaduras" en un 5,42\%; "No disponible" en un $49,82 \%$. Conclusión: Los datos indican que existe un problema relacionado con cuestiones técnicas y burocráticas con respecto a las recetas que interfieren con el acceso a los medicamentos.

Palabras clave: Servicios farmacéuticos, Medicamentos esenciales, Accesibilidad a los Servicios de Salud, Atención primaria de salud, Sistema único de salud.

\section{INTRODUÇÃO}

A Organização Mundial da Saúde (OMS) define saúde como "um estado de completo bem-estar físico, mental e social e não somente ausência de afecções e enfermidades". Nesse contexto as ações de Assistência Farmacêutica (AF) podem ser entendidas como condutas voltadas à promoção, proteção e recuperação da saúde, tanto individual como coletiva, tendo o medicamento como elemento essencial e visando o acesso e ao seu uso racional (DISTRITO FEDERAL, 2020; BRASIL, 2004).

Os medicamentos contribuem, em muitos casos, de forma decisiva para o controle das doenças e o aumento da expectativa de vida da população, sendo que sua ausência ou o uso irracional expõe os usuários a uma série de riscos, como o maior agravamento da enfermidade como consequência do tratamento inefetivo, o surgimento de novos agravos e eventos adversos em função da insegurança da farmacoterapia, a ampliação do número de hospitalizações, entre outros (BRASIL, 2014b). O acesso a tais insumos é reconhecido pela Organizações das Nações Unidas como um dos cinco indicadores que mensuram os avanços na garantia dos direitos à saúde (HOGERZEIL HV e MIRZA Z, 2011).

Diante dessa perspectiva, a AF, no âmbito do Sistema Único de Saúde (SUS) e enquanto parte integrante da Política Nacional de Saúde (PNS), visa à garantia do acesso a medicamentos e a insumos para toda população (BRASIL, 2018). A Lei 8.080/1990 institucionalizou a garantia do acesso a medicamentos no Brasil quando em seu Artigo 6엄 determinou como campo de atuação do SUS a:

"(...) formulação da política de medicamentos (...)" e atribui ao setor saúde a responsabilidade pela "execução de ações de assistência terapêutica integral, inclusive farmacêutica" (BRASIL, 1990).

As ações do estado brasileiro para promover acesso e qualificação da área de medicamentos no país remontam a um período pré-SUS, com a criação da Central de Medicamentos (CEME) em 1971. Este órgão foi planejado com o intuito de promover o acesso a medicamentos para populações mais pobres e fomentar a internalização da produção pública de medicamentos no País (BRASIL, 2002). A CEME vigorou entre 1971 e 1997, consistindo um marco importante no cenário de promoção do acesso a medicamentos (BRASIL, 2020b).

Com a desativação da CEME, o Ministério da Saúde formulou, de forma amplamente participativa, a Política Nacional de Medicamentos (PNM), aprovada pela Portaria $n^{\circ} 3.916$ em 1998. Esse importante documento visa a garantia da segurança, da eficácia e da qualidade dos medicamentos, além da promoção do uso racional e do acesso da população àqueles considerados essenciais (BRASIL, 1998a; VASCONCELOS DMM, et al., 2017). 
Ainda no final dos anos de 1990, a Política de Medicamentos Genéricos também contribui com o acesso a medicamentos, estimulando a variedade de oferta de tecnolocias em saúde com preços de maior acessibilidade (BRASIL, 1999a).

Somando-se a esse contexto, a necessidade de uma construção mais ampla e participativa da AF enquanto política pública, no início dos anos 2000, realizou-se a $1^{\text {a }}$ Conferência Nacional de Medicamentos e Assistência Farmacêutica (CNMAF), que teve como tema "Acesso, Qualidade e Humanização da Assistência Farmacêutica com Controle Social". Como resultado das deliberações desse processo de participação social, foi criada a Política Nacional de Assistência Farmacêutica (PNAF), aprovada pela Resolução № 338, de 6 de maio de 2004, do Conselho Nacional de Saúde, que efetivou a AF como política de saúde (BRASIL, 2004; BRASIL, 2018).

A garantia de acesso a medicamentos é particularmente importante no âmbito da Atenção Primária em Saúde (APS), que se caracteriza como porta de entrada preferencial no SUS, e é parte integrante do processo de promoção, recuperação e prevenção de doenças importantes do ponto de vista epidemiológico e social (ÁLVARES J, et al., 2017). Segundo a Política Nacional de Atenção Básica, é necessário desenvolver as ações de AF e de uso racional de medicamentos, garantindo a disponibilidade e o acesso a medicamentos e insumos (BRASIL, 2017).

O acesso a medicamentos do Componente Básico da Assistência Farmacêutica (CBAF) se dá por meio do atendimento nas Unidades Básicas de Saúde (UBS) do município onde reside o usuário. Entretanto, 0 processo é dificultado muitas vezes por questões burocráticas e também por desinformação tanto dos prescritores quanto dos usuários. Prescrições em desacordo com a legislação e a falta de documentos necessários para a dispensação são exemplos de fatores que impossibilitam o acesso, prejudicando o cuidado em saúde do usuário (BRASIL, 2007).

Diante do exposto, o objetivo do presente trabalho foi identificar as principais causas de demanda reprimida referentes ao acesso a medicamentos, considerado as barreiras documentais e/ou legais, em uma UBS do Distrito Federal, Brasil.

\section{MÉTODOS}

Foi realizado um estudo observacional, transversal, para a identificação das impossibilidades de acesso a medicamentos relacionadas a barreiras técnicas/legais. Os dados foram coletados de forma presencial por uma pesquisadora que acompanhou o serviço da dispensação durante os meses de julho e agosto de 2020. A coleta de dados ocorreu somente durante o período matutino em oito dias escolhidos aleatoriamente.

Durante os meses da coleta de dados, foram atendidas 6.581 prescrições em julho e 6.680 em agosto. No mês de julho a média foi de 286,13 prescrições atendidas por dia e no mês de agosto esse número subiu para 318,09. A amostra foi obtida por conveniência, pois a população de prescrições em que houve algum tipo de demanda reprimida ainda não era estimada numericamente. Durante os dias e período dispostos para a coleta de dados, foram captadas todas as prescrições que se enquadravam no objetivo do estudo. $O$ estudo foi realizado em uma UBS do Distrito Federal que funciona de segunda a sexta-feira de 07:00 às 18:00 horas.

Para a definição dos critérios de identificação das prescrições em que houve alguma impossibilidade de acesso, foi considerada a Portaria $n^{\circ} 250$, de 17 de Dezembro de 2014, que regula a prescrição e 0 fornecimento de medicamentos e produtos para a saúde no âmbito da Secretaria de Estado de Saúde do Distrito Federal (SES-DF) (DISTRITO FEDERAL, 2014). Tal portaria estabelece, dentre outras coisas, que para que o usuário tenha acesso a medicamentos do Componente Básico da AF é necessário apresentar prescrição médica em duas vias, documento de identificação e cartão SUS ou identificação/inscriçao da SES/DF do paciente.

Portanto, em consonância com a Portaria $n^{\circ}$ 250/2014, os critérios considerados para a identificação das barreiras de acesso foram: 1) Prescrições com informações incompletas, ilegíveis, com caracterização de emendas e/ou rasuras; 2) Prescrições com validade expirada; 3) Prescrições com medicamentos que não são previstos na Relação de Medicamentos do Distrito Federal (REME-DF); 4) Prescrições que não estão de acordo com a Denominação Comum Brasileira (DCB); 5) Ausência de duas vias (original e cópia); 6) Ausência de documentos indicados na portaria para a dispensação. 
Para as prescrições de medicamentos sob Regime de Controle especial, assim como dita 0 artigo $7^{\circ}$ da Portaria 250/2014, foram seguidas as normas definidas pela Portaria SVS/MS n 344, de 12 de maio de 1998 e suas atualizações (BRASIL, 1998a). Para as prescrições de antimicrobianos, foram seguidas as recomendações da Resolução da Diretoria Colegiada (RDC) nº 20 de 2011 da Agência Nacional de Vigilância Sanitária (ANVISA), que dispõe sobre o controle de medicamentos à base de substâncias classificadas como antimicrobianos de uso sob prescrição, isoladas ou em associação (BRASIL, 2011).

Além dos critérios descritos previamente, também foi incluído a opção "outros" para aquelas situações em que a impossibilidade de acesso não se enquadrou em nenhum outro critério citado anteriormente.

As prescrições também foram avaliadas quanto a origem (público ou privado), a proveniência (Distrito Federal ou outra Unidade Federativa) e a categoria do profissional prescritor. Para identificação da origem e proveniência foi considerado o cabeçalho do receituário e para identificação da categoria profissional foi considerado o carimbo do prescritor. Foi excluído do estudo a barreira de acesso referente ao desabastecimento de tecnologias na unidade visitada.

Para operacionalizar a coleta de dados foi utilizado um instrumento criado pelos próprios autores. Para melhor organização das informações coletadas, os dados foram transferidos para o Programa Exce| ${ }^{\circledR}$ na versão do ano 2013. Na sequência, os dados da planilha foram importados para o software IBM SPSS ${ }^{\circledR}$, onde foi feita a caracterização descritiva da análise.

A pesquisa foi aprovada pelo Comitê de Ética em Pesquisa da Fundação de Ensino e Pesquisa em Ciências da Saúde (Parecer 3.805.441). Essa investigação foi realizada de forma a garantir o sigilo dos dados coletados, assegurando a privacidade e a confidencialidade das informações dos usuários e dos prescritores.

Não foram coletadas informações sociodemográficas dos usuários, sendo coletadas somente informações técnicas e referentes aos medicamentos das prescrições. Ademais, considerando que o estudo realizado não estabeleceu contato direto com os usuários, o Termo de Consentimento Livre e Esclarecido (TCLE) não foi necessário.

\section{RESULTADOS}

Durante o período de coleta de dados, foram identificadas 277 impossibilidades de acesso pelos critérios definidos em 254 prescrições. Desta forma, em $8,66 \%$ das prescrições $(n=22)$ foram identificados mais de um critério de impossibilidade de acesso.

Da população de 254 prescrições analisadas, a média de medicamentos prescritos foi de 3,28 medicamentos por prescrição. Em contrapartida, nesse mesmo grupo em análise, a média de medicamentos não fornecidos por alguma barreira de acesso foi de aproximadamente 1,54. Foram prescritos 834 medicamentos e não fornecidos 391 . Dessa maneira, $46,88 \%$ dos medicamentos prescritos não foram fornecidos. Em outras palavras a cada 10 medicamentos prescritos, 4,68 não foram fornecidos devido a alguma impossibilidade referente a barreiras técnicas/legais.

A Tabela 1 apresenta os resultados referentes a impossibilidade de acesso de acordo com os critérios definidos. É observado que que a impossibilidade de acesso "não disponibilizados" é responsável por quase $50 \%$ dos casos de não fornecimento.

Tabela 1 - Total de impossibilidades de acesso em uma UBS do Distrito Federal nos meses de Julho e Agosto de 2020.

\begin{tabular}{lcc}
\hline Motivo da impossibilidade & $\mathbf{N}$ & $\%$ \\
\hline Não disponibilizados & 138 & $49,82 \%$ \\
Ausência de duas vias & 39 & $14,08 \%$ \\
Em desacordo com a DCB & 27 & $9,75 \%$ \\
Ausência de documentos necessários & 17 & $6,14 \%$ \\
Informações incompletas, ilegíveis ou caracterização de emendas e rasuras & 15 & $5,42 \%$ \\
Validade expirada & 12 & $4,33 \%$ \\
Outros & 29 & $10,47 \%$ \\
\hline Total & $\mathbf{2 7 7}$ & $\mathbf{1 0 0 \%}$ \\
\hline
\end{tabular}

Fonte: Silva MFM, et al., 2021. 
$\mathrm{Na}$ categoria "Não disponibilizados", os medicamentos que apareceram mais de uma vez foram Metformina/Glifage ${ }^{\circledR} 500 \mathrm{mg}$ (36; 26,09\%), Atenolol $25 \mathrm{mg}$ (14; 10,14\%), Dipirona $500 \mathrm{mg}$ comprimido e Enalapril $10 \mathrm{mg}(6 ; 4,35 \%)$, Paracetamol $750 \mathrm{mg}(5 ; 3,62 \%)$, Dramin ${ }^{\circledR}$, Domperidona, Diazepam $10 \mathrm{mg}$, Nimesulida, Citaneurin ${ }^{\circledR}$, Trazodona e Zolpidem (2; 1,45\%). Na categoria "Em desacordo com a DCB", os medicamentos que apareceram mais de uma vez foram Aradois $^{\circledR} 50 \mathrm{mg}$ (Losartana) $(4 ; 14,81 \%)$, Puran T4 ${ }^{\circledR}$ 50 mcg (Levotiroxina) (3; 11,11\%), Glifage ${ }^{\circledR} 850 \mathrm{mg}$ (Metformina) $(3 ; 11,11 \%)$ Diamicron ${ }^{\circledR} 60 \mathrm{mg}$ (Gliclazida) (2; 7,41\%), Selozok ${ }^{\circledR} 50 \mathrm{mg}$ (Metoprolol) $(2 ; 7,41 \%)$.

$\mathrm{Na}$ impossibilidade de acesso por "Ausência de documentos necessários" foi verificado que $88,24 \%$ do não fornecimento foi referente a medicamentos de controle especial e $11,76 \%$ foi referente a insumos para 0 tratamento de diabetes e asma, como seringas, tiras para controle glicêmico e espaçador para uso de aerosol. Das 12 prescrições em que houve impossibilidade pela razão "Validade Expirada", 41,66\% são referentes a medicamentos de controle especial.Na categoria "Outros" observou-se razões distintas que não se enquadravam nos demais motivos de impossibilidade de acesso. A ausência do profissional farmacêutico por motivo de férias, impossibilitando a dispensação de medicamentos de controle especial, foi a razão mais frequente $(31,03 \%)$.

$\mathrm{Na}$ Tabela 2 é observado que mais de $90 \%$ da origem das prescrições são provenientes de entidades públicas e que cerca de $9 \%$ das prescrições foram produzidas em serviços privados de saúde.

Tabela 2 - Origem das prescrições que apresentaram alguma impossibilidade de acesso nos meses de julho e agosto de 2020.

\begin{tabular}{lcc}
\hline Origem das Prescrições & $\mathbf{N}$ & $\%$ \\
\hline Público & 230 & $90,55 \%$ \\
Privado & 24 & $9,45 \%$ \\
Total & $\mathbf{2 5 4}$ & $\mathbf{1 0 0} \%$ \\
\hline
\end{tabular}

Fonte: Silva MFM, et al., 2020.

$\mathrm{Na}$ Tabela 3 é observado que o médico foi o principal profissional prescritor e que houve apenas duas categorias profissionais entre os prescritores, médico e enfermeiro. Somente os profissionais legalmente habilitados, cumprindo com os códigos de seus devidos conselhos, podem realizar prescrições de medicamentos.

Tabela 3 - Quantidade de prescrição por categoria profissional que apresentaram alguma impossibilidade de acesso nos meses de Julho e Agosto de 2020.

\begin{tabular}{lcc}
\hline Categoria Profissional & $\mathbf{N}$ & $\%$ \\
\hline Médico & 247 & $97,24 \%$ \\
Enfermeiro & 7 & $2,76 \%$ \\
Total & $\mathbf{2 5 4}$ & $\mathbf{1 0 0 \%}$ \\
\hline
\end{tabular}

Fonte: Silva MFM, et al., 2020.

Em relação à Unidade Federativa, foi observado que apenas uma prescrição foi proveniente de outro estado, sendo inviável para a análise inferencial.

\section{DISCUSSÃO}

$\mathrm{O}$ acesso à medicamentos essenciais, aqueles que atendem às necessidades epidemiológicas e sociais de uma população, constitui uns dos eixos norteadores das políticas de medicamentos e de AF (BRASIL, 1998b; BRASIL, 2004). Desta maneira, a ideia é justamente fornecer subsídios para melhor atender as necessidades da terapêutica e racionalizar o tratamento e não o de restringir a oferta. Há décadas, o Brasil vem investindo na publicação e no aperfeiçoamento de listas de medicamentos essenciais, consistindo estratégias fundamentais para garantia do acesso e do uso racional de tecnologias farmacêuticas (BRASIL, 2020c).

Desde a implantação da PNM, foi estabelecida a reorientação da AF, direcionada para o seu intensivo processo de descentralização. Dentro desse contexto está inserido o Componente Básico da Assistência 
Farmacêutica (CBAF), que é constituído por uma relação de medicamentos e insumos farmacêuticos, voltados aos agravos prevalentes e prioritários da Atenção Básica, presentes nos anexos I e IV da Relação Nacional de Medicamentos (RENAME) (OLIVEIRA LCF, et al., 2010).

De acordo com os resultados encontrados nessa investigação, o principal motivo observado do não fornecimento foi a prescrição de medicamentos não disponibilizados pela REME-DF (49,82\%). Apesar deste estudo não avaliar a variável desabastecimento, este resultado corrobora com o apresentado pela Pesquisa Nacional sobre o Acesso, Utilização e Promoção do Uso Racional de Medicamentos (PNAUM) no Brasil de que a dimensão "disponibilidade" ainda é reconhecida como a principal barreira de acesso no Brasil (ÁLVARES J, et al., 2017). Um estudo realizado na cidade de Blumenau/SC no ano de 2010 avaliou algumas dimensões do acesso na APS. Neste estudo também foi verificado que a maior parte dos motivos do não acesso a medicamentos (42,5\%) se deve à prescrição daqueles não fornecidos pelo SUS (HELENA ETS, et al., 2015).

Dentre os medicamentos que apareceram mais de uma vez na categoria "Não Disponibilizados", três deles (Metformina $500 \mathrm{mg}$, Atenolol $25 \mathrm{mg}$ e Enalapril $10 \mathrm{mg}$ ) são fornecidos pelo Programa Farmácia Popular do Brasil (PFPB).

O Programa implantado em 2004 possui como objetivo principal a ampliação do acesso a medicamentos e, inicialmente, tinha como público alvo indivíduos de baixa renda que utilizam serviços privados de saúde, e que têm dificuldades em adquirir medicamentos de que necessitam em farmácias privadas (BRASIL, 2005). Entretanto, o programa é utilizado atualmente de forma ampla pelos usuários do SUS como forma de garantir aqueles medicamentos que não são disponibilizados, mas que fornecidos pelo Programa Farmácia Popular do Brasil (PFPB).

Contudo, apesar dos usuários possuírem esta alternativa para o acesso, a maior parte dos medicamentos não fornecidos pela impossibilidade "Não disponibilizados" também não são fornecidos pelo PFPB. A escolha do medicamento é uma prerrogativa do prescritor, porém ações educativas de divulgação/valorização dos medicamentos essenciais voltadas a esses profissionais devem ser estimuladas como parte da estratégia para melhorar o acesso (BRASIL, 2006).

Ainda sobre os medicamentos que apareceram mais de uma vez na categoria "Não disponibilizados", foi identificado o medicamento Diazepam na concentração de $10 \mathrm{mg}$. Tal medicamento é disponibilizado pela SES/DF através da APS, porém em concentração de $5 \mathrm{mg}$. Dessa forma, é importante que o prescritor esteja familiarizado com a Relação de Medicamentos disponíveis no município/Distrito e suas devidas concentrações.

A impossibilidade de acesso por "Ausência de duas vias" é verificada como a segunda causa de demanda reprimida (14,08\%). A portaria que regula o fornecimento de medicamentos no âmbito do Distrito Federal estabelece que para todadispensação, uma via deve ficar retida na farmácia (DISTRITO FEDERAL, 2014). Essa ação visa, além do cumprimento das questões normativas, o levantamento de informações necessárias para etapas técnico-logísticas, para a rastreabilidade dos resultados do serviço de dispensação e para outros motivos de interesse da gestão da AF.

Foi observado que a maior parte dos medicamentos não fornecidos em razão de "Ausência de duas vias" são de uso contínuo, ou seja, são aqueles em que o paciente tem que ir mensalmente à unidade e deixar retida uma cópia impressa da prescrição original para o recebimento. Isso pode ser justificado pelo fato de, prescrições de medicamentos de uso pontual e de controle especial poderem ser atendidas somente uma vez, em que uma das vias é retida na farmácia no ato da dispensação. Desta maneira, não é possível a retirada de um novo medicamento mediante a apresentão de uma cópia, sendo necessário a apresentção de uma nova prescrição.

As prescrições em consoância com a DCB, além de serem uma exigência da SES-DF, é também prevista na Lei Federal ํㅜ 9.787, de 10 de fevereiro de 1999 que dispõe em seu artigo $3^{\circ}$ que as aquisições de medicamentos, sob qualquer modalidade de compra, e as prescrições médicas e odontológicas de medicamentos, no âmbito do Sistema Único de Saúde, adotarão obrigatoriamente a DCB ou, na sua falta, a Denominação Comum Internacional (DCI) (BRASIL, 1999b). 
Essas informações são relevates pelo fato de que mais da metade das prescrições $(66,66 \%)$ em que não houve o fornecimento pela razão da não prescrição em DCB ser proveniente de profissionais que atendem em serviços públicos. Portanto é importante destacar que, embora haja iniciativas de capacitação e fomento voltadas à prescrição de medicamentos segundo a DCB no SUS, ainda é grande a falta de adesão a esses pressupostos pelos prescritores.

O não fornecimento por razões que não se encaixavam nas demais categorias foram classificadas como "Outros". Foram identificados distintos motivos, sendo a ausência do profissional farmacêutico (impossibilitando a acesso a medicamentos de controle especial), a razão mais recorrente $(31,03 \%)$.

A Resolução $n^{\circ}$ 357/2001 do Conselho Federal de Farmácia (CFF) estabelece que a dispensação das substâncias e medicamentos sujeitos a controle especial deverá ser feita exclusivamente por farmacêutico, sendo vedado delegar a responsabilidade sobre a chave dos armários a outros funcionários da farmácia que não sejam farmacêuticos (BRASIL, 2001) .Desta maneira, durante o período de ausência do farmacêutico fica comprometido o fornecimento de medicamentos de controle especial, gerando prejuízos para o paciente, que precisa recorrer a outra UBS ou aguardar o retorno do profissional.

Enquanto o farmacêutico se encontrar ausente, seria de competência da SES-DF a disponibilização de outro profissional com a mesma formação/cargo, pois é sabido que, para o funcionamento das farmácias de qualquer natureza, é necessário ter a presença de farmacêutico durante todo o horário de funcionamento (BRASIL, 2014a).

Ainda na categoria "Outros", outro motivo bastante recorrente foi prescrição de medicamentos em que a via de acesso não se processa por meio de unidade da APS (24,14\%). No âmbito da SES-DF, o acesso a alguns medicamentos como Quetiapina e Risperidona ocorre por meio dos Núcleos de Farmácia do Componente Especializado (NFCE), conhecidas popurlamente como "farmácias de alto custo".

Para que o usuário tenha acesso a medicamentos disponibilizados pelos Componente Especializado da Assistência Farmacêutica, é necessário seguir um fluxo específico como agendamento de atendimento em uma unidade dispensadora e apresentação de um Laudo de Solicitação de Medicamentos (DISTRITO FEDERAL, 2018). Desta maneira, o acesso a esses recursos é dificultado porque muitos usuários e também profissionais prescritores não estão familiarizados com esse processo.

A impossibilidade de acesso por "Ausência de documentos necessários" só ocorreu em prescrições de medicamentos de controle especial. É estabelecido em portaria que, para o fornecimento de qualquer medicamento junto às farmácias das UBS do Distrito Federal, o usuário ou seu representante deverá apresentar além do Cartão Nacional de Saúde (CNS) ou Identificação SES/DF do paciente, o documento de identificação do paciente com foto (DISTRITO FEDERAL, 2014).

Entretanto, na prática dos serviços farmacêuticos da UBS em que o estudo foi realizado, para medicamentos não controlados, o fornecimento era realizado somente com a apresentação do CNS ou Identificação SES/DF. Isso pode justificar o motivo do não fornecimento por ausência de documentos ocorrer somente em medicamentos controlados. Porém, não podemos desconsiderar as questões burocráticas/técnicas que envolvem a dispensação desses insumos.

Para a dispensação de medicamentos de controle especial, é necessário o preenchimento com informações do comprador (no caso o indivíduo que irá retirar o medicamento), além de documento de identificação do mesmo (BRASIL, 1998b). Contudo, é verificado que isso geralmente é motivo de confusão, em que quando um representante vai retirar o medicamento para o paciente é levado somente o documento do paciente e não do individuo que está retirando. O mesmo ocorre com o preenchimento da prescrição. Essas questões técnicas também prejudicam o acesso do medicamento ao usuário.

A impossibilidade de acesso pelo motivo de "Validade expirada" foi a menos recorrente $(4,33 \%)$. No âmbito da SES-DF prescrições de medicamentos de uso contínuo possuem validade de 180 dias. Essa prática visa garantir a revisão da prescrição de medicamentos dos pacientes pelos prescritores ao longo do tempo. Entretanto, em função da pandemia de COVID-19, que foi justamente o momento em que esse trabalho foi desenvolvido, o tempo de validez das prescrições foi aumentado. Portanto, esse fato pode ser uma justificativa para a menor ocorrência de impossibilidade em razão de prescrições vencidas (BRASIL, 2020a). 


\section{CONCLUSÃO}

A avaliação de acesso a medicamentos é complexa e ainda é um grande desafio. A precariedade dos serviços do SUS aliada a uma extensa burocracia podem ser fatores que distanciam ainda mais o usuário do direito ao acesso a medicamentos. Os resultados apresentados por esse estudo indicam que existe um problema relacionado a questões técnicas e burocráticas referentes as prescrições que interferem no acesso a medicamentos.Certamente, são limitações desse estudo a investigação ter ocorrido somente em uma UBS e por curto período de tempo, além de ter utilizada uma amostra de conveniência no recrutamento das prescrições, o que pode gerar um viés de seleção. Contudo, acredita-se que a discussão levantada por esse estudo possibilita o aprofundamento do conhecimento acadêmico em uma área pouco explorada pela literatura científica e espera-se que investigações futuras sejam realizadas sobre o tema.

\section{REFERÊNCIAS}

1. ÁlVARES J, et al. Acesso aos medicamentos pelos usuários da atenção primária no Sistema único de Saúde. Revista de Saúde Pública, 2017; 51(2).

2. BRASIL. Conselho Federal de Farmácia. Resolução $n^{\circ} 357$ de 20 de Abril de 2001. Aprova o regulamento técnico das Boas Práticas de Farmácia. Diário Oficial da União. 27 de abr. de 2001. Disponível em: https://www.cff.org.br/userfiles/file/resolucoes/357.pdf. Acessado em: 01 de maio de 2021.

3. BRASIL. Conselho Nacional de Secretários de Saúde. Assistência Farmacêutica no SUS. 2007. Disponível em: https://bvsms.saude.gov.br/bvs/publicacoes/colec_progestores_livro7.pdf. Acessado em: 27 de maio de 2021.

4. BRASIL. Lei $n^{\circ} 13.021$, de 8 de agosto de 2014. Dispõe sobre o exercício e a fiscalização das atividades farmacêuticas. Diário Oficial da União. 11 de ago. de 2014a. Disponível em: http://www.planalto.gov.br/ccivil_03/_ato2011-2014/2014/lei/l13021.htm. Acessado em: 29 de abril de 2021.

5. BRASIL. Lei $n^{\circ} 14.028$, de 27 de julho de 2020. Altera a Lei $n^{\circ} 13.979$, de 6 de fevereiro de 2020, para garantir que o receituário médico ou odontologico de medicamentos sujeitos a prescrição e de uso contínuo tenha validade pelo menos enquanto perdurarem as medidas de isolamento para contenção do surto de Covid-19, na forma de especificada. Diário Oficional da União. 28 de jul. de 2020a. Disponível em: http://www.planalto.gov.br/ccivil_03/_ato2019-2022/2020/lei/L14028.htm. Acessado em: 10 de maio de 2021.

6. BRASIL. Lei $n^{\circ} 9.787$, de 10 de fevereiro de 1999. Altera a Lei $n^{\circ} 6.360$, de 23 de setembro de 1976, que dispõe sobre a vigilância sanitária, estabelece o medicamento genérico, dispõe sobre a utilização de nomes genéricos em produtos farmacêuticos e dá outras providências. Diário Oficial da União. 11 de fev. de 1999a. Disponível em: http://www.planalto.gov.br/ccivil_03/leis/l9787.htm\#: :text=LEl\%20N\%C2\%BA\%209.787\%2C\%20DE\%2010\%20DE \%20FEVEREIRO\%20DE\%201999.\&text=Altera\%20a\%20Lei\%20no,farmac\%C3\%AAuticos\%20e\%20d\%C3\%A1\% 20outras\%20provid\%C3\%AAncias. Acessado em: 23 de maio de 2021.

7. BRASIL. Lei no 9.787, de 10 de fevereiro de 1999. Altera a Lei no 6.360, de 23 de setembro de 1976, que dispõe sobre a vigilância sanitária, estabelece o medicamento genérico, dispõe sobre a utilização de nomes genéricos em produtos farmacêuticos e dá outras providências. Diário Oficial da União. 11 de fev. de 1999. Disponível em: http://www.planalto.gov.br/ccivil_03/leis/19787.htm. Acessado em: 10 de maio de 2021.

8. BRASIL. Lei 8.080, de 19 de setembro de 1990. Dispõe sobre as condições para a promoção, proteção e recuperação da saúde, a organização e o funcionamento dos serviços correspondentes e dá outras providências. Diário Oficial da União. 19 set. de 1990. Disponível em: http://www.planalto.gov.br/ccivil_03/leis/l8080.htm. Acessado em: 12 de maio de 2021.

9. BRASIL. Ministério da Saúde, Secretaria de Ciência, Tecnologia e Insumos Estratégicos. Departamento de Assistência Farmacêutica e Insumos Estratégicos Assistência Farmacêutica no SUS: 20 anos de políticas e propostas para desenvolvimento e qualificação: relatório com análise e recomendações de gestores, especialistas e $\begin{array}{lllll}\text { representantes da } & \text { sociedade } & \text { civi. } & \text { Disponível }\end{array}$ http://bvsms.saude.gov.br/bvs/publicacoes/assistencia_farmaceutica_sus_relatorio_recomendacoes.pdf. Acessado em: 30 de abril de 2021.

10. BRASIL. Ministério da Saúde. Agência Nacional de Vigilância Sanitária. Resolução da Diretoria Colegiada nº 20 , de 5 de maio de 2011. Dispõe sobre o controle dos medicamentos à base de substâncias antimicrobianas. Diário Oficial da União. 9 de maio. 2011.2 Disponível em: https://bvsms.saude.gov.br/bvs/saudelegis/anvisa/2011/rdc0020_05_05_2011.html. Acessado em: 02 de maio de 2021.

11. BRASIL. Ministério da Saúde. Conselho Nacional de Saúde. Resolução no 338, de 6 de maio de 2004. Aprova a política nacional de assistência farmacêutica. Diário Oficial da União. 20 de mai. de 2004. Disponível em: http://bvsms.saude.gov.br/bvs/saudelegis/cns/2004/res0338_06_05_2004.html. Acessado em: 02 de maio de 2021.

12. BRASIL. Ministério da Saúde. Cuidado Farmacêutico na Atenção Básica. Serviços Farmacêuticos na Atenção Básica. 2014b. Disponível em: https://bvsms.saude.gov.br/bvs/publicacoes/servicos_farmaceuticos_atencao_basica_saude.pdf. Acessado em: 13 de maio de 2021. 
13. BRASIL. Ministério da Saúde. Departamento de Assistência Farmacêutica e Insumos - DAF. Assistência Farmacêutica na Atenção Básica. 2006. Disponível em: http://www.ensp.fiocruz.br/portalensp/judicializacao/pdfs/283.pdf. Acessado em: 13 de maio de 2021.

14. BRASIL. Ministério da Saúde. Fundação Oswaldo Cruz. Programa Farmácia Popular do Brasil. 2005. Dispoível em: http://bvsms.saude.gov.br/bvs/publicacoes/PROGRAMA_FARMACIA_POPULAR.pdf. Acessado em: 12 de maio de 2021.

15. BRASIL. Ministério da Saúde. Fundação Oswaldo Cruz. Desafios do Acesso a Medicamentos no Brasil. $2020 \mathrm{~b}$. Disponível em: https://www.arca.fiocruz.br/bitstream/icict/41803/2/Desafios_do_Acesso_a_Medicamentos_2020.pdf. Acessado em: 06 de maio de 2021.

16. BRASIL. Ministério da Saúde. Política Federal de Assistência Farmacêutica 1990 a 2002. 2002. Disponível em: http://bvsms.saude.gov.br/bvs/publicacoes/polit_fed_assist_farm.pdf. Acessado em 10 de maio de 2021.

17. BRASIL. Ministério da Saúde. Portaria $n^{\circ}=2.436$, de 21 de setembro de 2017 . Aprova a política nacional de atenção básica, estabelecendo a revisão de diretrizes para a organização da atenção básica, no âmbito do sistema único de saúde (SUS). Diário Oficial da União. 22 de set. de 2017. Disponível em: https://bvsms.saude.gov.br/bvs/saudelegis/gm/2017/prt2436_22_09_2017.html. Acessado em: 13 de maio de 2020.

18. BRASIL. Ministério da Saúde. Portaria no. 3.916, de 30 de outubro de 1998. Aprova a Política Nacional de Medicamentos. Diário Oficial da União. 10 de nov. de 1998a. Disponível em: https://bvsms.saude.gov.br/bvs/saudelegis/gm/1998/prt3916_30_10_1998.html. Acessado em: 04 de maio de 2021.

19. BRASIL. Ministério da Saúde. Secretaria de Ciência, Tecnologia, Inovação e Insumos Estratégicos em Saúde. Departamento de Assistência Farmacêutica e Insumos Estratégicos. Relação Nacional de Medicamentos Essenciais

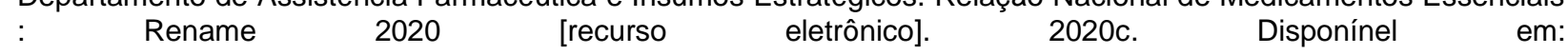
https://bvsms.saude.gov.br/bvs/publicacoes/relacao_medicamentos_renam e_2020.pdf. 2020b. Acessado em 01 de dezembro de 2020.

20. BRASIL. Ministério da Saúde. Secretaria de Vigilância em Saúde. Portaria nํ344, de 12 de maio de 1998. Aprova o regulamento técnico sobre substâncias e medicamentos sujeitos a controle especial. Diário Oficial da União. 31 de dez. de 1998b. Disponível em: https://bvsms.saude.gov.br/bvs/saudelegis/svs/1998/prt0344_12_05_1998_rep.html. Acessado em 12 de maio de 2021.

21. DISTRITO FEDERAL. Secretaria de Estado de Saúde do Distrito Federal. Guia de Boas Práticas para Serviços Farmacêuticos desenvolvidos no ambiente hospitalar. 2020. Disponível em: http://www.saude.df.gov.br/wpconteudo/uploads/2018/04/GUIA_Boas_Praticas_Farma\%CC\%81cia_Hospitalar_SES_DF_-_maio2020_finalizado.pdf. Acessado em: 12 de maio de 2021.

22. DISTRITO FEDERAL. Secretaria de Estado de Saúde do Distrito Federal. Portaria $n^{\circ} 250$, de 17 de dezembro de 2014. Dispõe sobre normas técnicas e administrativas relacionadas à prescrição e fornecimento de medicamentos e produtos para a saúde da Assistência Farmacêutica Básica, no âmbito da Secretaria de Estado de Saúde do Distrito Federal. Diário Oficial do Distrito Federal. 17 de dez. de 2014 . Disponível em: http://www.tc.df.gov.br/sinj/Norma/78835/Portaria_250_17_12_2014.html. Acessado em 11 de maio de 2021.

23. DISTRITO FEDERAL. Secretaria de Estado de Saúde do Distrito Federal. Componente Especializado da Assistência Farmacêutica. 2018. Disponível em: http://www.saude.df.gov.br/componente-especializado. Acessado em: 21 de janeiro de 2021.

24. HELENA ETS, et al. Percepção dos usuários sobre acesso aos medicamentos na atenção primária. Caderno Saúde Coletiva, 2015; 23(3): 280-288.

25. HOGERZEIL HV, MIRZA Z. The world medicines situation 2011: access to essential medicines as part of the right to health. World Health Organization, 2011; $3^{\text {a }}$ ed.

26. OLIVEIRA LCF, et al. Assistência Farmacêutica no Sistema Único de Saúde: da Política Nacional de Medicamentos à Atenção Básica à Saúde. Ciência e Saúde Coletiva, 2010; 15(3): 3561-3567.

27. VASCONCELOS DMM, et al. Política Nacional de Medicamentos em retrospectiva: um balanço de (quase) 20 anos de implementação. Ciência e Saúde Coletiva, 2017; 22(8): 2609 - 2614. 